\title{
Hiper-homocisteinemia associada ao aumento no risco de doença vascular obstrutiva periférica: verdade ou mito?
}

\author{
Hyperhomocysteinemia associated with an increased risk for peripheral \\ arterial disease: truth or myth?
}

\begin{abstract}
Nelson Wolosker*
A aterotrombose é a maior causa de mortalidade no hemisfério norte, sendo responsável por $54 \%$ dessa estatística ${ }^{1}$. Em nosso país, ela também é a maior causa de mortalidade $^{2}$, sendo a doença arterial obstrutiva periférica (DAOP) uma de suas principais manifestações clínicas ${ }^{3}$.

A DAOP é uma doença sistêmica que se desenvolve com o passar dos anos e permanece assintomática na maior parte da vida (fase assintomática). Quando sintomática, pode levar a limitações de marcha (claudicação intermitente $)^{4,5}$ ou a situações onde a demanda de oxigênio e nutrientes não seja suficiente para manter o metabolismo basal dos tecidos dos membros inferiores (isquemia crítica) ${ }^{6}$.

A busca pelos mecanismos geradores da DAOP vem estimulando os pesquisadores a investigarem fatores de risco específicos. Diversos fatores já foram definidos e muito bem estudados em trabalhos clínicos e experimentais. Por exemplo, hoje não existem dúvidas quanto ao papel do diabetes melito (DM), do tabagismo, da hipercolesterolemia e dos fatores hereditários na geração da DAOP. Na busca por melhores resultados no tratamento da DAOP, o conhecimento de fatores preditivos permite que tenhamos uma ideia do prognóstico de cada caso ${ }^{6-8}$.

Há 40 anos, McCully observou que crianças com maiores níveis séricos de homocisteína de causa genética apresentavam aterosclerose prematura e propôs que esse aminoácido levava a aterotrombose arterial e venosa ${ }^{9}$.

A homocisteína é um aminoácido produzido pelo catabolismo da metionina ou da cisteína, presente em todos

os indivíduos e elevada em certas condições. Estudos experimentais confirmaram que a lesão vascular é associada à exposição a altas concentrações desse catabólito, sendo que elas ocorrem tanto por estresse oxidativo, lesão endotelial, disfunção endotelial, inflamação, trombose e proliferação celular ${ }^{10}$.

Muitos trabalhos comprovaram a associação entre a hiper-homocisteinemia e o aumento do risco para aterosclerose $^{10}$, trombose $^{11}$, acidente vascular cerebral ${ }^{12} \mathrm{e}$ $\mathrm{DAOP}^{13}$. Boushey et al. ${ }^{11}$ verificaram, em meta-análise, que níveis de homocisteína aumentados, de 3 a $4 \mu \mathrm{mol} / \mathrm{L}$, poderiam levar a um aumento de risco de doença cardiovascular de até $30 \%$. Nessa investigação, os autores analisaram 27 estudos, correlacionando níveis séricos de homocisteína e doença aterosclerótica, e realizaram metaanálise em cinco deles, demonstrando associação entre níveis elevados de homocisteína e DAOP. O estudo foi atualizado este ano por Khandanpour et al. ${ }^{14}$, que analisaram 14 artigos relevantes, demonstrando que a homocisteína estava significativamente elevada em pacientes com DAOP em relação ao grupo controle.

Robinson et al. ${ }^{15}$ realizaram estudo multicêntrico em pacientes com DAOP, doença cerebrovascular e doença coronariana, e verificaram que as concentrações de homocisteína no plasma superiores ao percentil 80 estavam associadas a aumento no risco de DAOP, independentemente da presença de DM, tabagismo, hipertensão arterial e histoplasmose pulmonar crônica.
\end{abstract}

\footnotetext{
* Professor associado, Faculdade de Medicina, Universidade de São Paulo (USP), São Paulo, SP.

Não foram declarados conflitos de interesse associados à publicação deste editorial. 
Bergmark et al. verificaram que o aumento dos níveis de homocisteína está associado ao aumento da extensão da DAOP, visto que os níveis da homocisteína eram menores em pacientes com doença em um único nível em relação àqueles com doença em vários níveis ${ }^{16}$. Frier et al. observaram que o aumento nos níveis de homocisteína está relacionado ao aparecimento de sintomas em indivíduos mais jovens ${ }^{17}$.

A maioria das meta-análises e também dos estudos caso-controle multicêntricos foi realizada em países do hemisfério norte. Dessa forma, é muito importante saber se esses achados são reprodutíveis no Brasil. Venâncio et al. ${ }^{18}$, do grupo de Botucatu, apresentam neste número do J Vasc Bras um ensaio clínico caso-controle muito interessante, comparando um grupo de pacientes portadores de DAOP confirmado por Doppler ultrassom a um grupo de indivíduos livres dessa doença selecionado em um serviço público brasileiro. Os autores confirmaram os achados internacionais e forneceram dados objetivos sobre as concentrações plasmáticas de homocisteína de jejum. Observaram que pacientes com DAOP apresentavam níveis médios de $16,7 \mu \mathrm{mol} / \mathrm{L}$, enquanto que pacientes normais apresentavam níveis médios de homocisteína de 12,9 $\mu \mathrm{mol} / \mathrm{L}$. A partir de agora, podemos nos basear nesses dados para orientar pacientes brasileiros quanto à melhor forma de tratamento.

Níveis plasmáticos de homocisteína são influenciados por fatores genéticos, que são imutáveis, mas também por níveis plasmáticos de ácido fólico e vitamina $\mathrm{B} 12^{19}$. Diversos estudos demonstram que, em alguns casos, os níveis de homocisteína podem ser diminuídos por suplementação dessas duas substâncias, diminuindo o risco cardiovascular ${ }^{20}$.

Mais de 20.000 pacientes já foram incluídos em clinical trials para o estudo da relação entre suplementação alimentar (ácido fólico e vitamina B12) e doença coronária ou cerebral. Entretanto, apenas 290 pacientes com DAOP foram investigados de forma consistente. Essa falta de conhecimento gera uma oportunidade científica que deve ser estimulada em nossas instituições de pesquisa, que poderão aumentar o conhecimento desse interessante e importante tema ${ }^{14}$.

\section{Referências}

1. Belch JJ, Topol EJ, Agnelli G, Bertrand M, Califf RM, Clement DL. Prevention of atherothrombotic disease network. Critical issues in peripheral arterial disease detection and management: a call to action. Arch Intern Med. 2003;163:884-92.

2. de Souza MF, Alencar AP, Malta DC, Moura L, Mansur AP. Serial temporal analysis of ischemic heart disease and stroke death risk in five regions of Brazil from 1981 to 2001. Arq Bras Cardiol. 2006;87:735-40.

3. Wolosker N, Nakano L, Rosoky RA, Puech-Leão P. Evaluation of walking capacity over time in 500 patients with intermittent claudication who underwent clinical treatment. Arch Intern Med. 2003;163:2296-300.

4. Ayzin Rosoky RM, Wolosker N, Muraco-Netto B, PuechLeão P. Ground reaction force pattern in limbs with intermittent claudication. Eur J Vasc Endovasc Surg. 2000;20:254-9.

5. Ferrari FB, Wolosker N, Rosoky RA, D'Ippolito G, Wolosker AM, Puech-Leão P. Natural history of stenosis in the iliac arteries in patients with intermittent claudication undergoing clinical treatment. Rev Hosp Clin Fac Med Sao Paulo. 2004;59:341-8.

6. Wolosker N, Muraco Neto B, Munia MA, Rosoky RA, Ramos RS, Puech-Leão P. External aggression to the limb as a predictive factor in the evolution of patients undergoing arterial revascularization. Clinics. 2005;60:451-4.

7. Wolosker N, Rosoky RA, Nakano L, Basyches M, PuechLeão P. Predictive value of the ankle-brachial index in the evaluation of intermittent claudication. Rev Hosp Clin Fac Med Sao Paulo. 2000;55:61-4.

8. Nasser M, Wolosker N, Uint L, et al. Relationship between soluble thrombomodulin in patients with intermittent claudication and critical ischemia. Thromb Res. 2006;117:271-7.

9. McCully KS. Vascular pathology of homocystinemia. Am J Pathol. 1969;56:111-28.

10. Welch GN, Loscalzo J. Homocysteine and atherothrombosis. N Engl J Med. 1998;338:1042-50.

11. Boushey CJ, Beresford SA, Omenn GS, Motulsky AG. A quantitative assessment of plasma homocysteine as a risk factor for vascular disease. JAMA. 1995;274:1049-57.

12. The Homocysteine Studies Collaboration. Homocysteine and risk of ischemic heart disease and stroke. JAMA. 2002;288:2015-22.

13. Eikelboom JW, Lonn E, Genest J, Hankey G, Yusuf S. Homocyst(e)ine and cardiovascular disease. Ann Intern Med. 1999;131:363-75.

14. Khandanpour N, Loke YK, Meyer FJ, Jennings B, Armon MP. Homocysteine and peripheral arterial disease: systematic review and meta-analysis. Eur J Vasc Endovasc Surg. 2009;38:316-22.

15. Robinson K, Arheart K, Refsum H, et al. Low circulating folate and vitamin B6 concentrations: risk factors for stroke, peripheral vascular disease, and coronary artery disease. European COMAC Group. Circulation. 1998;97:437-43.

16. Bergmark C, Mansoor MA, Swedenborg J, de Faire U, AM Svardal, Ueland PM. Hyperhomocysteinemia in patients operated for lower extremity ischaemia below the age of 50: effect of smoking and extent of disease. Eur J Vasc Surg. 1993;7;391-6.

17. Fryer RH, Wilson BD, Gubler DB, Fitzgerald LA, Rodgers GM. Homocysteine, a risk factor for premature vascular disease and thrombosis, induces tissue factor activity in endothelial cells. Arterioscler Thromb. 1993;13:1327-33. 
18. Venâncio LS, Burini RC, Yoshida WB. Concentração de homocisteína em pacientes portadores de doença arterial periférica atendidos em um serviço público de saúde. J Vasc Bras. 2009;8:313-321.
19. Wang X, Qin X, Demirtas H, et al. Efficacy of folic acid supplementation in stroke prevention: a meta-analysis. Lancet. 2007;369:1876-82.

20. Homocysteine Lowering Trialists' Collaboration. Dosedependent effects of folic acid on blood concentrations of homocysteine. Am J Clin Nutr. 2005;82:806-12. 